\title{
Status of the PANDA Endcap Disc DIRC project
}

\author{
I. Köseoglu, ${ }^{a, e, 1}$ A. Ali, ${ }^{a, b}$ A. Belias, ${ }^{a}$ R. Dzhygadlo, ${ }^{a}$ A. Gerhardt, ${ }^{a}$ M. Krebs, ${ }^{a, b}$ \\ D. Lehmann, ${ }^{a}$ K. Peters, ${ }^{a}, b$ G. Schepers, ${ }^{a}$ C. Schwarz, ${ }^{a}$ J. Schwiening, ${ }^{a}$ M. Traxler, ${ }^{a}$ \\ L. Schmitt, ${ }^{c}$ M. Böhm, ${ }^{d}$ A. Lehmann, ${ }^{d}$ M. Pfaffinger, ${ }^{d}$ S. Stelter, ${ }^{d}$ F. Uhlig, ${ }^{d}$ M. Düren, ${ }^{e}$ \\ E. Etzelmüller, ${ }^{e}$ K. Föhl, ${ }^{e}$ A. Hayrapetyan, ${ }^{e}$ K. Kreutzfeldt, ${ }^{e}$ J. Rieke, ${ }^{e}$ M. Schmidt, ${ }^{e}$ \\ T. Wasem ${ }^{e}$ and C. Sfienti ${ }^{f}$
}

${ }^{a}$ GSI Helmholtzzentrum für Schwerionenforschung GmbH, Darmstadt, Germany

${ }^{b}$ Goethe University, Frankfurt a.M., Germany

${ }^{c}$ FAIR, Facility for Antiproton and Ion Research in Europe, Darmstadt, Germany

${ }^{d}$ Friedrich Alexander University of Erlangen-Nuremberg, Erlangen, Germany

${ }^{e}$ II. Physikalisches Institut, Justus Liebig University of Giessen, Giessen, Germany

${ }^{f}$ Institut für Kernphysik, Johannes Gutenberg University of Mainz, Mainz, Germany

E-mail: ilknur.koeseoglu@exp2.physik.uni-giessen.de

Abstract: The PANDA experiment at FAIR near Darmstadt, Germany, is under construction, planned to investigate fundamental questions of hadron physics with a fixed proton target by using an antiproton beam within a momentum range of 1.5 to $15 \mathrm{GeV} / \mathrm{c}$. A novel Cherenkov detector, the Endcap Disc DIRC (EDD), has been developed to separate $\pi^{ \pm}$and $K^{ \pm}$by at least 3 standard deviation up to $4 \mathrm{GeV} / \mathrm{c}$. It will cover the polar angle range of the forward endcap of the PANDA target spectrometer from $5^{\circ}$ to $22^{\circ}$. The EDD uses a $2 \mathrm{~cm}$ thick fused silica radiator plate with focusing elements at the outer rim and lifetime-enhanced Microchannel-Plate PMTs (MCP-PMTs) as light sensors. The Cherenkov radiator of the EDD consists of four identical independent quadrants, polished with highest precision in order to conserve the internally reflected Cherenkov angle during light propagation. This manuscript confirms basic functionalities of the EDD design and tests of the prototype with particle beams at CERN in 2018.

Keywords: Cherenkov and transition radiation; Cherenkov detectors; Particle identification methods

${ }^{1}$ Corresponding author. 


\section{Contents}

1 Introduction 1

2 The Endcap Disc DIRC 1

2.1 Design of the EDD 1

2.2 Cherenkov radiation and reconstruction of the Cherenkov angle 2

2.3 Readout system 4

3 The EDD prototype experiments at CERN 5

$\begin{array}{lll}3.1 & \text { Time offset correction } & 6\end{array}$

$\begin{array}{lll}3.2 & \text { Testbeam results } & 7\end{array}$

4 Conclusion \& outlook $\quad 7$

\section{Introduction}

The fixed target experiment PANDA [2] at the future Facility of Antiproton and Ion Research (FAIR) in Darmstadt, Germany will investigate fundamental questions of hadron physics by using a cooled antiproton beam with a beam momentum range from $1.5 \mathrm{GeV} / \mathrm{c}$ to $15 \mathrm{GeV} / \mathrm{c}$. The PANDA detector is going to be implemented for the High Energy Storage Ring (HESR) that will provide the antiproton beam with two different modes of operation: a high resolution mode that will be applied for the starting phase of the PANDA and a high luminosity mode with the highest luminosity of $\mathcal{L}=2 \cdot 10^{32} \mathrm{~cm}^{-2} \mathrm{~s}^{-1}$.

The PANDA detector setup consists of two parts: the target spectrometer as an onion shell-like detector around the interaction point and the forward spectrometer, which is going to cover small polar angles up to $5^{\circ}$ in vertical and $10^{\circ}$ in the horizontal direction along the beam line.

In order to obtain an excellent identification of $\pi, K$ and $p$, two DIRC (Detection of Internally Reflected Cherenkov light) detectors are included in the target spectrometer of PANDA in figure 1 (left). The simulated kaon phase space is shown in the right plot of figure 1. The Barrel DIRC [9] will be built around the interaction point and provides 3 standard deviation (s.d.) separation up to $3.5 \mathrm{GeV} / \mathrm{c}$ in the range of polar angles from $22^{\circ}-140^{\circ}$. The Endcap Disc DIRC (EDD) will cover the forward region of the target spectrometer and provide 3 s.d. up to $4 \mathrm{GeV} / \mathrm{c}$ between $5^{\circ}$ and $22^{\circ}$.

\section{The Endcap Disc DIRC}

\subsection{Design of the EDD}

The EDD is a novel type of DIRC detector featuring a compact disc shape. The Cherenkov radiator of the EDD is a $2 \mathrm{~cm}$ thin plate of synthetic fused silica with a dodecagon shape divided into 4 identical quadrants. The outer radius of each quadrant is $1056 \mathrm{~mm}$. The quadrants will be stabilized in beam 


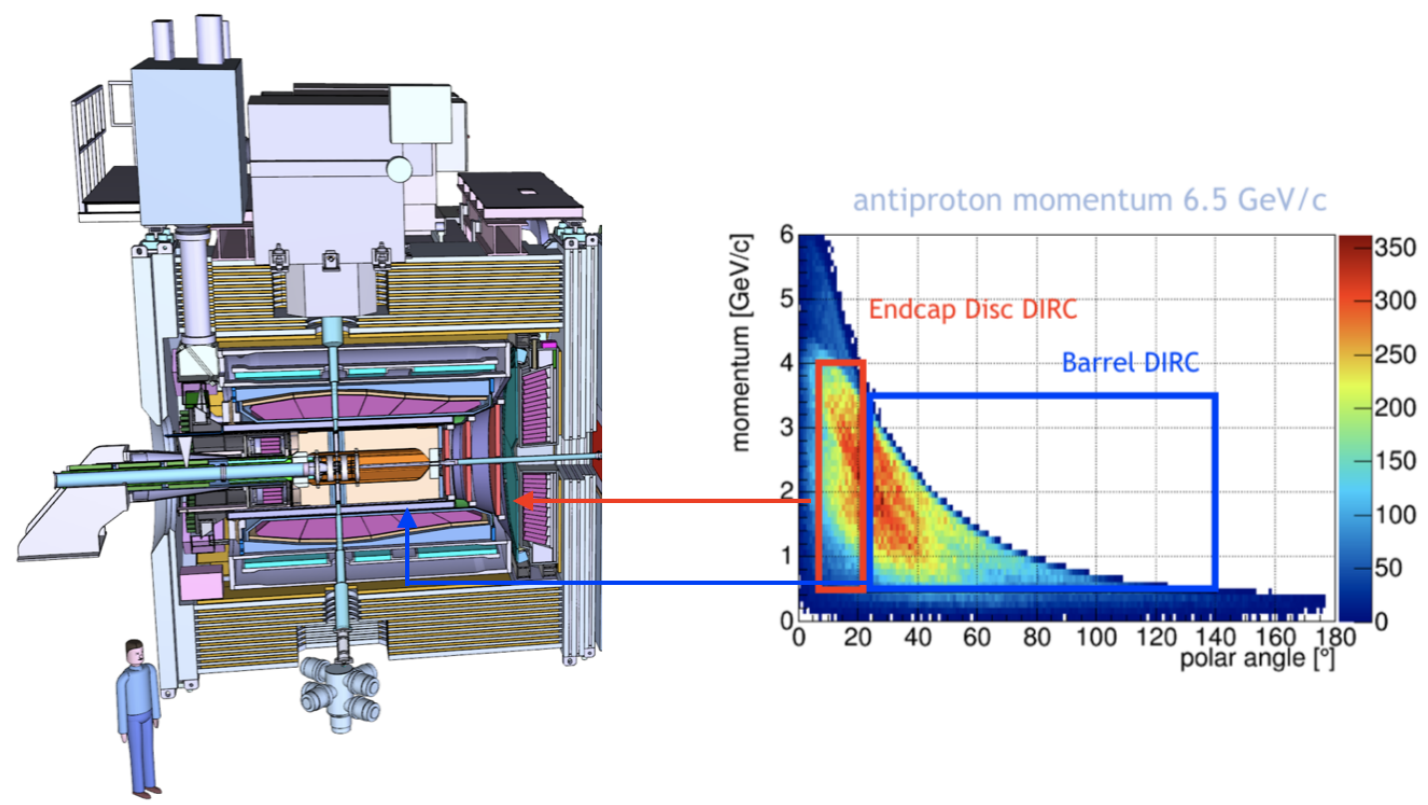

Figure 1. Left: the DIRC detectors in the PANDA target spectrometer. The antiproton beam is entering from the left side of the target spectrometer. The Barrel DIRC is going to cover polar angle from $22-140^{\circ}$ and the EDD will cover from $\left(5-22^{\circ}\right)$. Right: most of the simulated kaon phase space is covered by DIRCs.

direction by using a holding cross that will be mounted on a ring-shaped mounting frame [7]. The EDD and endcap EMC detectors are planned to be fixed opposite sides of the same holding frame. The mounting frame will be fixed on to an upstream side of the holding frame while the downstream side will be occupied by the endcap electromagnetic calorimeter (EMC). A CAD drawing including all elements of the EDD can be seen in figure 2. Each quadrant has 24 readout modules (ROM) with attached MCP-PMTs, 3 focusing elements (FELs) and expansion volumes with a cylindrical mirror at the backside. In total, 96 ROMs and 288 FELs will be used for the fully equipped detector. The bars are going to be glued to the radiator. Each ROM case will be light-tight to prevent external photon entry and also provide gas tightness, as a nitrogen gas flow will be used to keep the surfaces of the optical elements clean. An ASIC-based readout system will be mounted on the MCP-PMT. The analog signals will be digitized by ASICs and the digital signals will be transmitted to the data acquisition via a radiation-tolerant optical link.

\subsection{Cherenkov radiation and reconstruction of the Cherenkov angle}

The DIRC concept is visualized in figure 3 for the novel EDD [5]. From left to right: Cherenkov photons (purple) are emitted when a charged particle (green arrow) traverses the disc-shaped radiator with a speed that is faster than the speed of light in fused silica. Those photons will be internally reflected and propagated through the outer rim where ROMs are attached in figure 3 (center). The photons will be focused on the surface of the MCP-PMT [4] via a cylindrical mirror at the backside of the FEL. Analog signals will be digitized by an ASIC readout system and sent to the PANDA compute node via a bi-directional optical link. The hit pattern will be reconstructed with a fast geometric reconstruction algorithm to calculate the Cherenkov angle. 


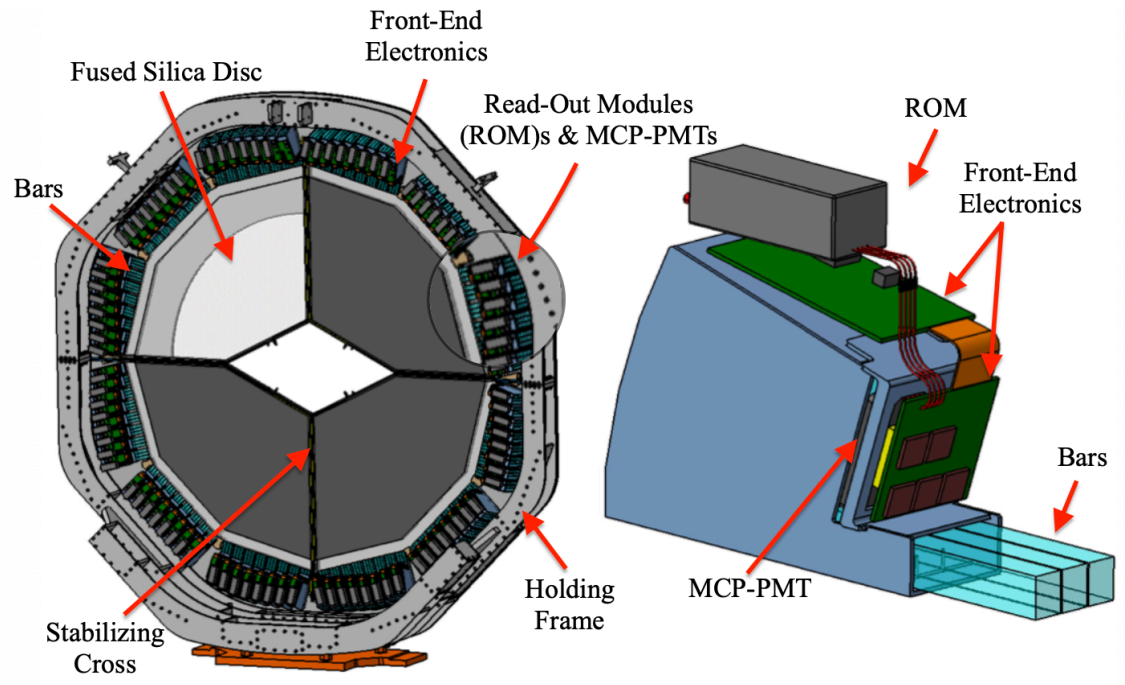

Figure 2. Left: the EDD with elements. 96 ROMs in total. Right: one ROM with front-end electronics.
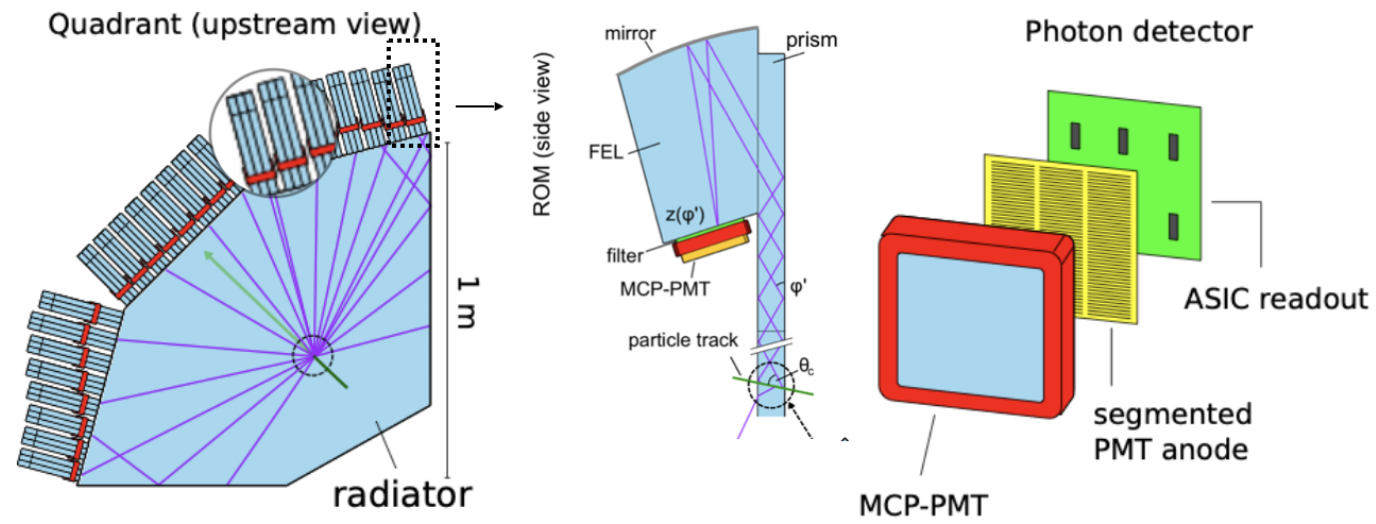

Figure 3. Left: quadrant upstream view with ROMs and MCP-PMTs. Right: each MCP-PMT consist of a highly segmented anode with $3 \times 100$ pixels. An ASIC read out board is connected to the MCP-PMT anode.

The definition of the geometric angles involved can be seen in figure 4. The angle between the charged particle and the photon is $\phi_{\text {rel }}$, the angle between the orientation of the FEL and the photon trajectory is $\alpha_{\mathrm{FEL}}$. In the side view of the radiator, the Cherenkov cone is represented as a reddish area with the Cherenkov angle $\theta_{c}$, and the magnitude of the $\varphi$ is conserved during the internal reflection. The $\varphi^{\prime}$ angle is dependent on the sensor position, which shows a linear behavior with respect to the z-coordinate of the focal plane of the sensor

$$
\varphi^{\prime}(z)=m z+b
$$

The relation between $\varphi^{\prime}$ and $\varphi$ can be calculated

$$
\tan \varphi^{\prime}=\frac{\tan \varphi}{\cos \alpha_{\mathrm{FEL}}}
$$



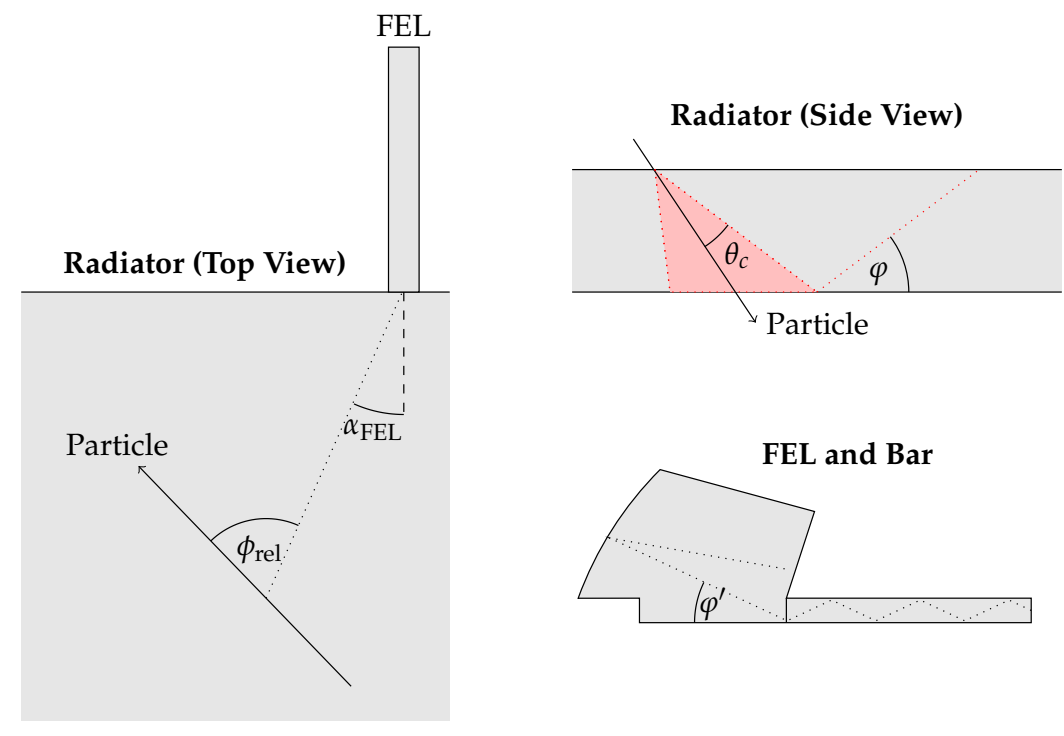

Figure 4. Angle definitions from the side and top view of the radiator [8].

The Cherenkov angle can be calculated analytically according to

$$
\theta_{c}=\arccos \left(\sin \theta_{P} \cos \phi_{\mathrm{rel}} \cos \varphi+\cos \theta_{P} \sin \phi\right),
$$

where $\theta_{P}$ is the polar angle of the charged particle.

In order to reconstruct the Cherenkov angle, the so-called backward method has to be applied for each photon hit by using

$$
\cos \theta_{c}=\frac{1}{n \beta}
$$

where the refractive index $n$ is obtained from the average wavelength, and $\beta$ is defined as

$$
\beta=\frac{p}{\sqrt{p^{2}+m^{2} c^{2}}}
$$

with $p$ as momentum of the beam.

\subsection{Readout system}

The readout system of the EDD is being developed by the company PETsys [6] which is specialized in the design of medical PET detectors. It will consist of a rigid-flex design with two rigid boards connected by one flex signal transmission board in the middle to save space. The rigid board with 5 TOFPET ASICs will be attached to the adapter board that includes routing 300 analog signals of the MCP-PMT. The MCP-PMTs will be powered by separated high voltage suppliers in combination with high voltage dividers. The fully equipped EDD detector will have 28800 channels that will be connected to the readout system. The analog signals are digitized by ASICs and transmitted via the flex PCB to the other rigid board that consists of an FPGA, a low voltage input and a bi-directional optical link. The bi-directional optical link is going to provide the clock and synchronization 
of the PANDA synchronization system (SODAnet). Digitized data are sent via the PANDA data concentrator to the PANDA data acquisition (DAQ) system [3]. Some of the electronic elements, such as ASICs, FPGA, and DC regulators will require a dedicated cooling system to dissipate their heat.

The readout system provides a $30 \mathrm{ps}$ time resolution and $3.2 \mathrm{~Gb} / \mathrm{s}$ of maximum output data rate. The maximum achievable data rate is approximately $100 \mathrm{kHz}$ per channel. In the high luminosity mode, the expected interaction rate is $20 \mathrm{MHz}$, which corresponds to a hit frequency of $60 \mathrm{kHz}$ per channel.

\section{The EDD prototype experiments at CERN}

Several experiments were carried out DESY and CERN with different prototypes. The test beam data was taken in the CERN PS/T9 area in August 2018. The beam contains a mixture of mainly pions and protons. The particle momentum varied between $3 \mathrm{GeV} / \mathrm{c}$ and $10 \mathrm{GeV} / \mathrm{c}$. The size of the radiator prototype is $500 \times 500 \mathrm{~mm}^{2}$ (figure 5) and the thickness is $20 \mathrm{~mm}$.

During the beam time, ROMs had been coupled to the radiator and MCP-PMTs (two Photonis MCP-PMTs with $3 \times 100$ channels and a Hamamatsu MCP-PMT with $6 \times 128$ channels) attached to the ROMs. Different position scans were done with various momenta, some of them are depicted in figure 5 with red dots. Figure 5 shows a sketch of the upstream view of the prototype: the red dots indicate the beam positions for the vertical scan and the point labeled with A highlights the position of the beam for different angles of the prototype. A PiLas laser is diffused into the prototype from the left side for the purpose of calibration.

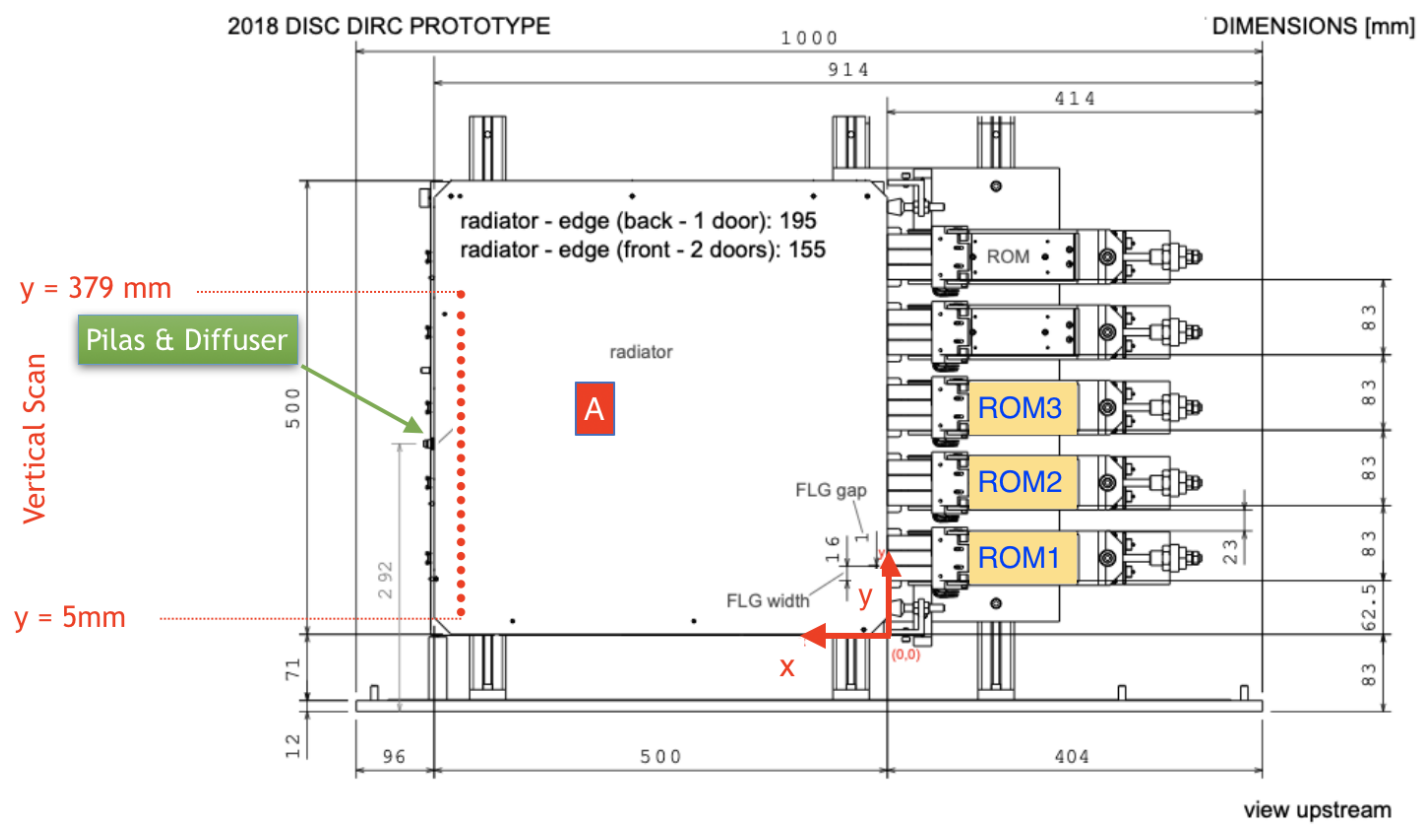

Figure 5. Disc DIRC prototype with dimensions. The red dots correspond to the beam positions. PiLas is a picosecond pulsed laser for calibration. 


\subsection{Time offset correction}

The readout system of the prototype consists of ASICs. In order to determine their signal timing offsets, the laser pulser was used as a reference signal. Each ROM has an MCP-PMT and each row of MCP-PMT corresponds to one focusing element. Because of the cable constraints, signals were obtained from 96 channels per MCP-PMT row.
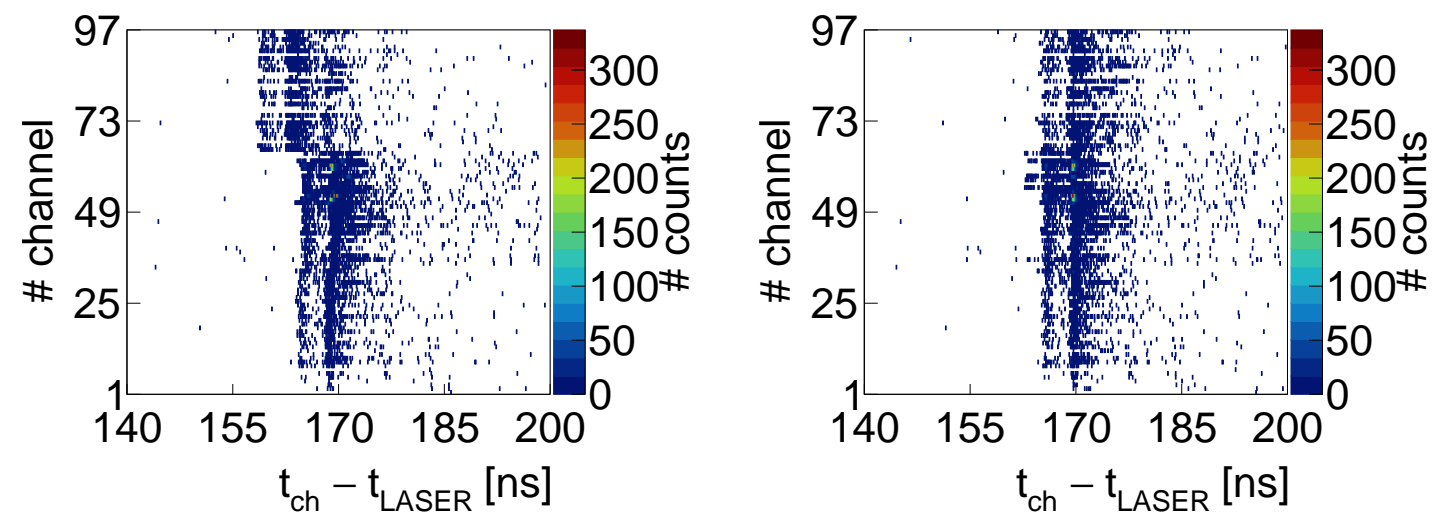

Figure 6. Left: number of hits per channel as a function of the time difference between the laser signal and the time of the signal, before correction. Right: channel hits as a function of time difference between laser signal and signal time, after correction.
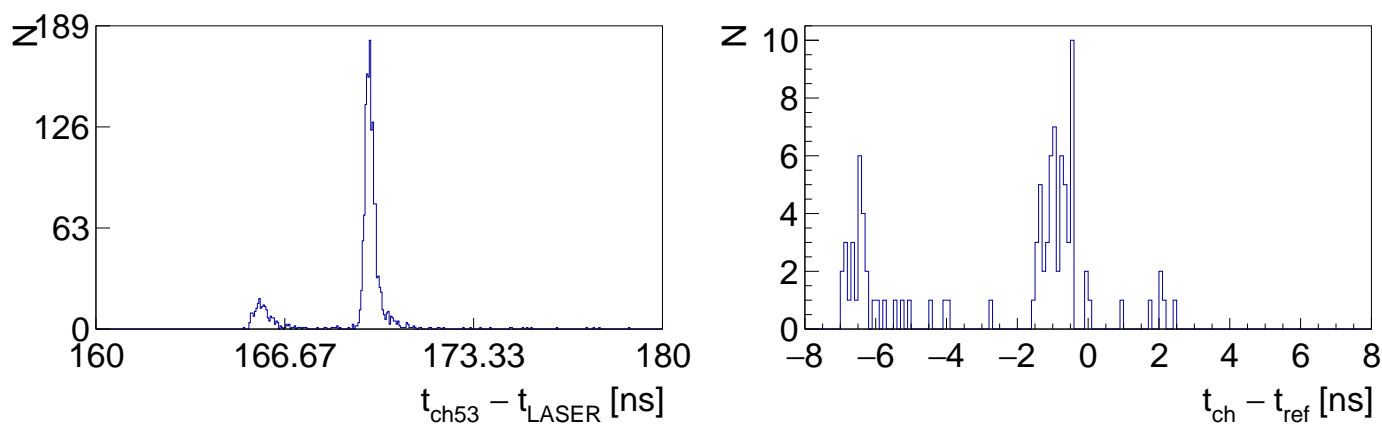

Figure 7. Left: the time difference between laser signal and reference channel. Right: time delays between the reference channel and the other channels.

Each ASIC has 64 channels and each row of the MCP-PMT was connected to two different ASICs. In order to eliminate the time difference between the ASICs (see figure 6), a time offset correction method had been applied for all ASICs.

In order to apply the method, the channel (channel 53) with a maximum number of hits was chosen (see figure 7, left) and its time difference with the laser signal was chosen as a reference time. Afterward, the time difference between the time of each channel and the reference time was calculated. The time delays between the reference channel and the others can be seen in figure 7 (right). The corrected signal alignment for all channels can be seen in figure 6 (right). 


\subsection{Testbeam results}

In the CERN PS/T9 area, two TOF counters were positioned $29 \mathrm{~m}$ apart from each other for separating pions and protons externally by their time-of-flight information at $7 \mathrm{GeV} / \mathrm{c}$ momentum.

During beam time, it turned out that the recent production of ASICs had problems with a negative signal polarity which led to problems during the calibration and threshold settings. As a result, fluctuating noise levels during beam time deteriorated our results by significantly reducing our readout efficiencies.
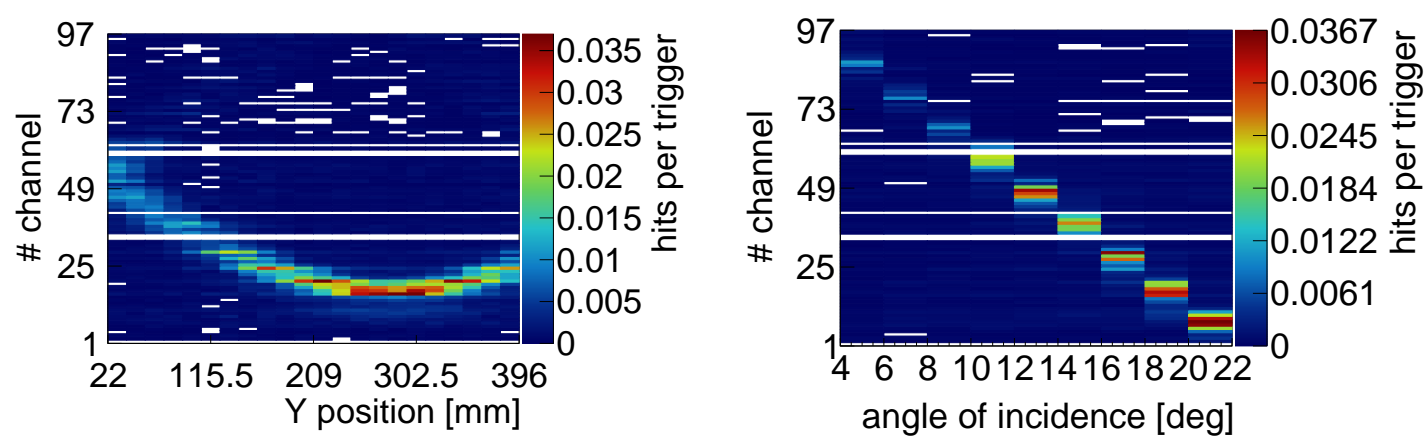

Figure 8. Left: channel number vs $\mathrm{Y}$ position at $7 \mathrm{GeV} / \mathrm{c}$ for pion. White lines represent missing channels. Right: channel distribution vs angle position at $7 \mathrm{GeV} / \mathrm{c}$ for pion.

Figure 8 shows the number of detected Cherenkov photons as a function of beam position and channel number at $7 \mathrm{GeV} / \mathrm{c}$ for pions. White lines correspond to the missing channels. The vertical axis represents the adjacent channels, the horizontal axis represents the positions (in $\mathrm{mm}$ or deg), and the z-axis represents the number of hits per trigger. The horizontal axis is changing from $22 \mathrm{~mm}$ to $379 \mathrm{~mm}\left(4^{\circ}\right.$ to $20^{\circ}$ ) for left (right) panel in figure 8 and the best coincidence region is between $200 \mathrm{~mm}$ and $370 \mathrm{~mm}$ in y position. Cherenkov angles were reconstructed for this region as shown in figure 9 (left). For the angle scan in figure 8 (right), the height of the entering beam is at $y=252 \mathrm{~mm}$. The reconstructed Cherenkov angle compared with Monte Carlo (MC) simulations for the vertical and the angle scans are shown in figure 9 for pions and protons. The reconstructed Cherenkov angles for separate particles show an agreement between data and MC simulations.

The reconstructed single photon resolution [mrad] for different positions in figure 10. MC results vary between $5-7 \mathrm{mrad}$ and data results vary between $5 \mathrm{mrad}$ and $10 \mathrm{mrad}$. The offset between the data and $\mathrm{MC}$ for lower and higher Y positions in figure 10 (left) can be explained as a result of the aforementioned readout problems. When the beam position gets larger, background dominants the signal that leads to worse single photon resolution (SPR) for data. Despite the polarity issues, one can say that data is an agreement with $\mathrm{MC}$ for both position scans.

\section{Conclusion \& outlook}

The Endcap Disc DIRC is one of the most important component of the PANDA experiment for particle identification. Testbeam data and MC show an expected agreement with each other, although there were some readout problems. The next generation of the ASICs of TOFPET will be 

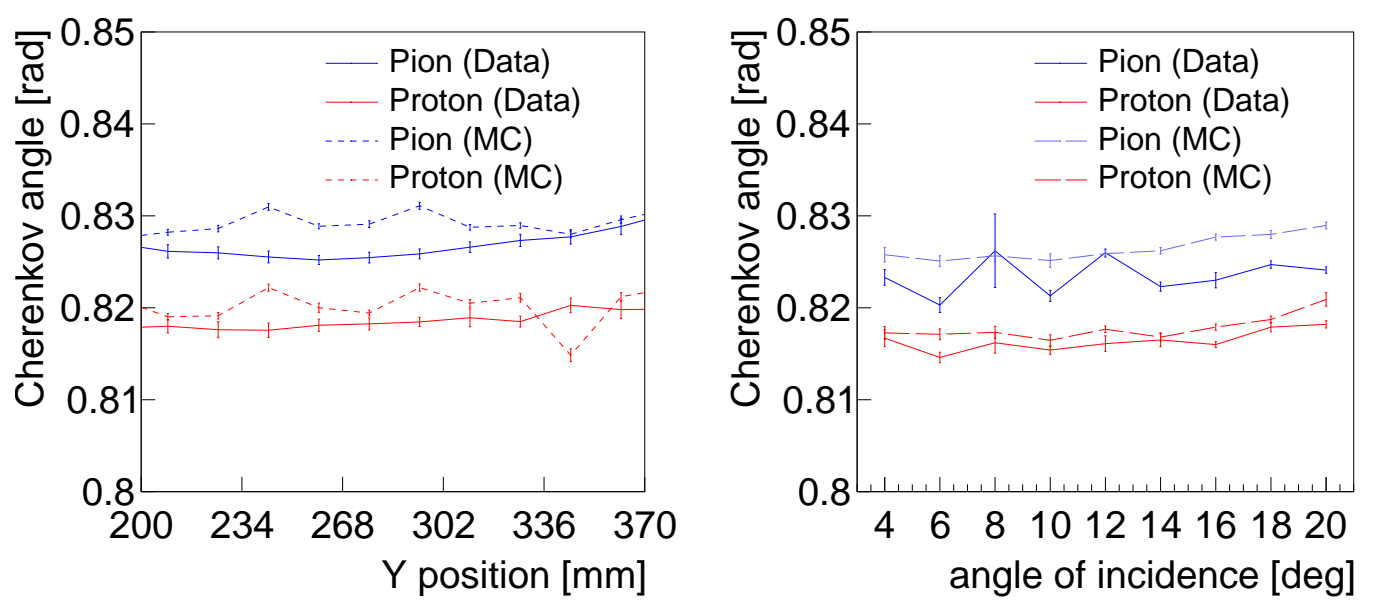

Figure 9. Left: reconstructed Cherenkov angle reconstruction for different vertical positions. Right: Cherenkov angle reconstruction for different angles of the incident beam.
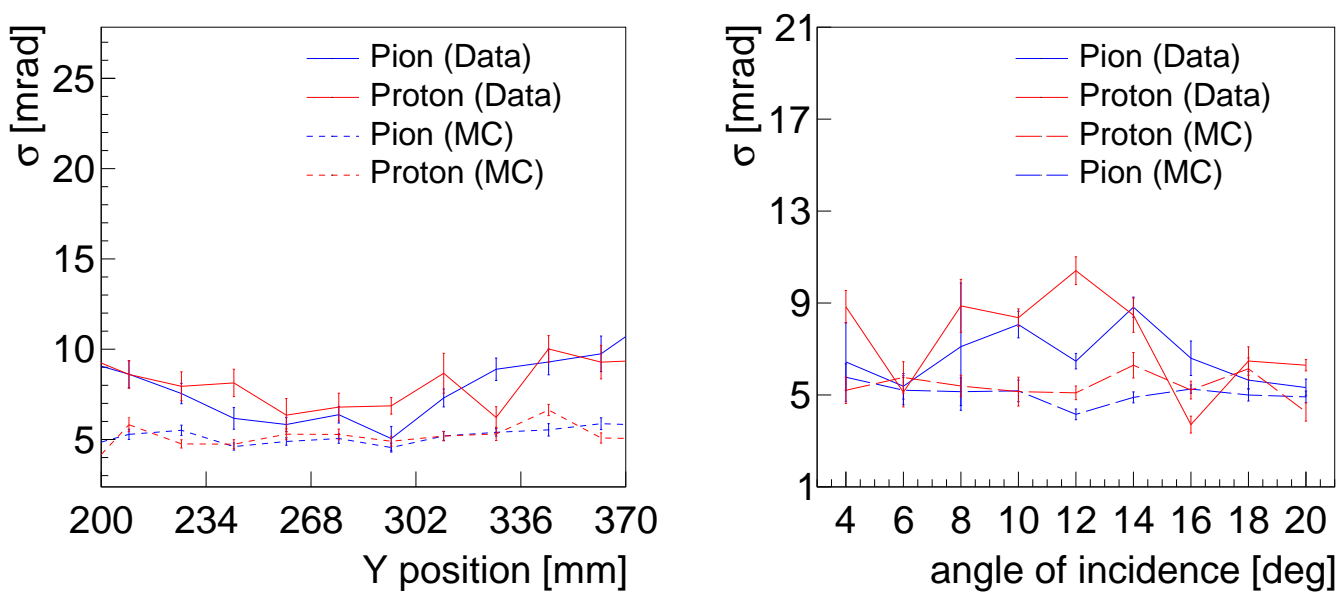

Figure 10. Single photon resolution for different vertical positions (Left) and different angle positions (Right).

compatible with both polarities. They are planned to be delivered at the beginning of 2020. Further measurements will be taken with new TOFPET ASICs in the Giessen Cosmic Station (GCS) [1].

The EDD component fabrication will start in 2021. The industrial fabrication of 4 independent quadrants, support frame, gluing FELs and detailed scans of all sensors are scheduled to be completed in 2025. The installation of the EDD in the PANDA target spectrometer is foreseen in 2026.

\section{References}

[1] S. Bodenschatz et al., The Giessen cosmics station, to be submitted to JINST (2020).

[2] PANDA collaboration, Technical Progress Report, FAIR-ESAC/Pbar 75 (2005). 
[3] W. Kühn et al., Data acquisition and online event selection for the PANDA experiment, JPS Conf. Proc. 18 (2017) 011035.

[4] A. Lehmann et al., Latest improvements of microchannel-plate PMTs. Nucl. Instrum. Meth. A (2019) 162357.

[5] O. Merle, Development, design and optimization of a novel Endcap DIRC for PANDA, Ph.D. thesis, Justus-Liebig-University, Gießen, Germania (2014).

[6] PETsys, PETsys Electronics, https://www.petsyselectronics.com/web/, (2020).

[7] M. Schmidt et al., The innovative design of the Endcap Disc DIRC Detector for PANDA at FAIR. in the proceedings of the Meeting of the Division of Particles and Fields of the American Physical Society (DPF2019), July 29-August 2, Boston, Massachusetts (2019).

[8] M.A. Schmidt, Particle identification with the Endcap Disc DIRC for PANDA, Ph.D. thesis, Justus-Liebig-University, Gießen, Germania (2017).

[9] B. Singh et al., Technical design report for the PANDA barrel DIRC detector, J. Phys. G 46 (2019) 045001. 